\title{
Isotropic Tensors Under Non-compact Rotation Groups
}

\author{
Ansaruddin Syed \\ Department of Mathematics, Jinah University for Women, Karachi, Pakistan
}

Email address:

ansaruddinsyed@gmail.com

\section{To cite this article:}

Ansaruddin Syed. Isotropic Tensors Under Non-compact Rotation Groups. American Journal of Applied Mathematics. Vol. 5, No. 2, 2017, pp. 39-47. doi: 10.11648/j.ajam.20170502.11

Received: October 21, 2016; Accepted: November 21, 2016; Published: May 27, 2017

\begin{abstract}
In the recent past, linearly independent isotropic tensors of rank up to 6, under the compact rotation groups $\mathrm{SO}(2)$, $\mathrm{SO}(3)$ and $\mathrm{SO}(4)$ have been studied in some detail. The present paper extends these studies to the case of linearly independent isotropic tensors under the non-compact rotation groups $\mathrm{SO}(1,1), \mathrm{SO}(1,2), \mathrm{SO}(1,3)$ and $\mathrm{SO}(2,2)$. This is done by using the direct method of explicitly constructing these tensors, proving their linear independence and counting their numbers. Interestingly, it is found that these numbers are identical with the corresponding numbers for the case of the compact groups $\mathrm{SO}(2), \mathrm{SO}(3)$ and $\mathrm{SO}(4)$.
\end{abstract}

Keywords: Isotropic Tensors, Linear Independence, Non-compact Rotation Groups

\section{Introduction}

Linear invariants and isotropic tensors of the rotation groups $\mathrm{SO}(2), \mathrm{SO}(3)$ and $\mathrm{SO}(4)$, have recently attracted the attention of many researchers. Thus Faiz and Riaz [1] have studied eigenvectors of isotropic tensors while Hirth and Lothe [2], Juret Schke [3], Hearrmon [4] and Norris [5] have considered the linear invariants of the stiffness tensor $\mathrm{C}_{\mathrm{ijk}}$, appearing in elasticity, under $\mathrm{SO}(3)$. Similarly Zhou et. al. [6], Lazar [7], Itin et. al. [8] and Gusev and Lurie [9] have used them in isotropic strain gradient elasticity theory. Next, Ahmad and Rashid [10] first showed that the number of linearly independent linear invariants of tensors of any rank under any rotation group $\mathrm{SO}(\mathrm{p})$, is equal to the dimension of the space of isotropic tensors of $\mathrm{SO}(\mathrm{p})$, of that rank, and then obtained all the linearly independent linear invariants under $\mathrm{SO}(2)$ of tensors up to rank 6 and those under $\mathrm{SO}(3)$ also up to rank 6. They also found the number of linearly independent linear invariants of a tensor of general rank, under the group of rotations about a fixed axis, say the 3axis. Recently, Naila Amir [11] has found the number of linearly independent isotropic tensors of ranks $r=2,4,6,8$, under $\mathrm{SO}(4)$ and has also obtained explicit expressions for complete sets of basis elements in the space of isotropic tensors of these ranks.

After having considered the isotropic tensors under these compact rotation groups, the question that immediately arises is: what happens when we move on to the non-compact rotation groups $\mathrm{SO}(1,1), \mathrm{SO}(2,1), \mathrm{SO}(3,1)$ and $\mathrm{SO}(2,2)$. This is the motivation for the present work in which, using the direct method, we explicitly construct a set of isotropic tensors, of rank up to 6 , under each of these non-compact groups, select a linearly independent subset of each of these sets, and then show that each of these selected subsets is complete in the sense that every isotropic tensor of the relevant type, is a linear combination of elements of this subset. This means that the current work is an immediate generalization of the work of Ahmad and Rashid [10] for $\mathrm{SO}(2)$ and $\mathrm{SO}(3)$ and of Naila Amir [11] for $\mathrm{SO}(4)$. An interesting fact which appears from this work, is that the number of elements of a complete set of linearly independent isotropic tensor of any particular type, under a non-compact group, is identical with the corresponding number, under the corresponding compact group. The basic reason for this appears to be the fact that the metric tensor $\delta_{\mathrm{ij}}$ of the Euclidean spaces and $\eta_{\mathrm{ij}}$ of the Minkowski spaces, both satisfy the identical relations

$$
\delta_{\mathrm{ij}}=0 \mathrm{i} \neq \mathrm{j} \text {, and } \eta_{\mathrm{ij}}=0, \mathrm{i} \neq \mathrm{j} .
$$

As in references $[10,11]$, we denote by $d$ the dimensions of the space in which rotations take place, and by $r$ the rank of the tensor. To proceed systematically, we start with the 
case $d=2$, and then move on to the higher values of $d=3,4$, one by one.

\section{2. $d$ = 2; Isotropic Tensors Under $\operatorname{SO}(1,1)$}

We take $\mathrm{SO}(1,1)$ to be the set of hyperbolic rotations (with determinant +1$)$ in the Minkowski space $M(1,1)$, with metric $\mathrm{x}^{2}=\mathrm{x}_{0}{ }^{2}-\mathrm{x}_{1}{ }^{2}$ i.e. the metric tensor $\eta_{\mathrm{ij}}, \mathrm{i}, \mathrm{j}=0,1$, is the matrix

$$
\eta=\left\{\eta_{i j}\right\}=\left[\begin{array}{cc}
1 & 0 \\
0 & -1
\end{array}\right]
$$

Then a $2 \times 2$ matrix a $=\left\{a_{i j}\right\}, \mathrm{i}, \mathrm{j}=0,1$, transforms a vector

$\mathrm{x} \varepsilon \mathrm{M}(1,1)$ according to the rule

$$
x_{i}^{\prime}=a_{i j} x_{j}
$$

and the condition for a to belong to $\mathrm{SO}(1,1)$ is

$$
a^{\mathrm{T}} \eta \mathrm{a}=\eta \Leftrightarrow \mathrm{a} \eta \mathrm{a}^{\mathrm{T}}=\eta
$$

as can be easily checked.

Note that (1) implies that a tensor

$$
T_{i_{1} i_{2} \ldots \ldots i_{k}}
$$

over $\mathrm{M}(1,1)$, transforms under a rotation $a \varepsilon \mathrm{SO}(1,1)$, to

$$
T_{i_{1} i_{2} \ldots \ldots i_{k}}^{\prime}=a_{i_{1} j_{1}} a_{i_{2 j_{2}}} \ldots \ldots a_{i_{k} j_{k}} T_{j_{1} j_{2} \ldots \ldots j_{k}},
$$

where Einstein's summation convention has been used. A tensor over $\mathrm{M}(1,1)$, is said to be ISOTROPIC if it has the same components in every coordinate system i.e. if

$$
T_{i_{1} i_{2} \ldots \ldots i_{k}}^{\prime}=T_{i_{1} i_{2} \ldots \ldots i_{k}}
$$

THE CASE $r=2$ :

It is a simple matter to check that the two second rank tensor

$$
\eta_{\mathrm{ij}}, \varepsilon_{\mathrm{ij}}
$$

are isotropic; here

$$
\varepsilon_{i_{1} i_{2} \ldots \ldots i_{k}}
$$

is the Levi-Civita symbol i.e. the completely anti-symmetric tensor of rank k. For

$$
\eta_{i j}^{\prime}=a_{i k} a_{j l} \eta_{k l}=a_{i k} \eta_{k l} a_{l j}^{T}=\left(\mathrm{a} \eta \mathrm{a}^{\mathrm{T}}{ }_{\mathrm{ij}}=\eta_{i j},\right.
$$

while isotropy of $\varepsilon_{\mathrm{i} j}$ follows from the easily verifiable fact that

$$
\epsilon_{i_{1} i_{2} \ldots i_{k}}
$$

is isotropic under $\mathrm{sl}(\mathrm{k}, \mathbb{R})$ (See Appendix A)

THE CASE $r=3$ :

As no tensors of rank 3 can be formed out of $\eta_{i j}$ and $\varepsilon_{i j}$ and $\varepsilon_{\mathrm{i} k \mathrm{k}}$ is identically zero for $\mathrm{d}=2$, it follows that there do not exist any isotropic tensors of rank 3 under $\mathrm{SO}(1,1)$; obviously, the same will be true for isotropic tensors of any ODD rank.

THE CASE $r=4$ :

Here the possible isotropic tensors, are

$$
\begin{gathered}
\eta_{i j} \eta_{k l}, \eta_{i k}, \eta_{i l} \eta_{j k}, \\
\epsilon_{i j} \epsilon_{k l}, \epsilon_{i k} \epsilon_{j l}, \epsilon_{i l} \epsilon_{j k}, \\
\eta_{i j} \epsilon_{k l}, \eta_{i k} \epsilon_{j l}, \eta_{j k} \epsilon_{i l}, \\
\eta_{i l} \epsilon_{j k}, \eta_{j l} \epsilon_{i k}, \eta_{k l} \epsilon_{i j} .
\end{gathered}
$$

However, not all of these are linearly independent as one can find a number of linear relations between them. To obtain these relations, one has to use the following result of Appendix B:

$$
=(-1)^{\mathrm{n}-1}\left|\begin{array}{c}
\epsilon_{i_{0} i_{1} \ldots i_{n-1}} \epsilon_{j_{0 j_{1} \ldots j_{n-1}}} \\
\eta_{i_{0 j_{0}}} \eta_{i_{0 j_{1}}} \ldots \eta_{i_{0} j_{n-1}} \\
\eta_{i_{1 j_{0}}} \eta_{i_{1 j_{1}}} \ldots \eta_{i_{1} j_{n-1}} \\
\cdot \\
\cdot \\
\eta_{i_{n-1 j_{0}}} \eta_{i_{n-1 j_{1}}} \ldots \eta_{i_{n-1} j_{n-1}}
\end{array}\right| .
$$

Taking $\mathrm{n}=2$, one gets

$$
\epsilon_{i j} \epsilon_{k l}=-\left|\begin{array}{ll}
\eta_{i j} & \eta_{i l} \\
\eta_{j k} & \eta_{j l}
\end{array}\right|=-\left(\eta_{i j} \eta_{j l}-\eta_{i l} \eta_{j k}\right) .
$$

One can similarly obtain expressions for

$$
\epsilon_{i k} \epsilon_{j l}, \epsilon_{i l} \epsilon_{j k}
$$

in terms of product of $2 \eta^{\mathrm{s}}$, so that we conclude that each of the 3 tensors (2.2) can be expressed as a linear combination of the 3 tensors (2.1).

Next, taking $n=3$ in (3), one gets

$$
\begin{gathered}
\epsilon_{i j k} \epsilon_{l m n}=\left|\begin{array}{lll}
\eta_{i l} & \eta_{i m} & \eta_{i n} \\
\eta_{j l} & \eta_{j m} & \eta_{j n} \\
\eta_{k l} & \eta_{k m} & \eta_{k n}
\end{array}\right| \\
=-\left(\eta_{i l} \epsilon_{j k} \epsilon_{m n}-\eta_{i m} \epsilon_{j k} \epsilon_{l n}+\eta_{i n} \epsilon_{j k} \epsilon_{l m}\right) \\
=-\epsilon_{j k}\left(\eta_{i l} \epsilon_{m n}-\eta_{i m} \epsilon_{l n}+\eta_{i n} \epsilon_{l m}\right) .
\end{gathered}
$$

As the LHS is zero for $\mathrm{d}=2$ and $\epsilon_{j k} \neq 0$, we get

$$
\eta_{i l} \epsilon_{m n}-\eta_{i m} \epsilon_{l n}+\eta_{i n} \epsilon_{l m}=0 .
$$

Choose now $\mathrm{i}=\mathrm{j}, \mathrm{m}=\mathrm{k}$ and then replace $\mathrm{n}$ by $\mathrm{l}$, to get

$$
\begin{gathered}
\eta_{i j} \epsilon_{k l}-\eta_{i k} \epsilon_{j l}+\eta_{i l} \epsilon_{j k}=0 \\
\Rightarrow \eta_{i l} \epsilon_{j k}=-\left(\eta_{i j} \epsilon_{k l}-\eta_{i k} \epsilon_{j l}\right) .
\end{gathered}
$$

This means that the first tensor of (2.4) is expressible as a linear combination of the 3 tensors (2.3); as the same will be true for the other 2 tensors of (2.4), it follows that we have proved, using an obvious notation, that

"All tensors of the form $\eta \epsilon_{\bullet j}$ and $\eta \epsilon_{\bullet k}$ have been 
expressed as a linear combination of tensors of the form $\eta \epsilon_{\bullet l} "$

It therefore follows that all the 12 isotropic tensors $(2.1$ 2.4) can be expressed in terms of 6 of them appearing in (2.1, 2.3). It is easy to check that these 6 tensors are linearly independent; for if

$$
\begin{gathered}
a_{1} \eta_{i j} \eta_{k l}+a_{2} \eta_{i k} \eta_{j l}+a_{3} \eta_{i l} \eta_{j k}+a_{4} \eta_{i j} \epsilon_{k l}+a_{5} \eta_{i k} \epsilon_{j l}+ \\
a_{6} \eta_{j k} \epsilon_{i l}=0
\end{gathered}
$$

then taking $\mathrm{i}=\mathrm{j}=0, \mathrm{k}=\mathrm{l}=1$, one gets $\mathrm{a}_{1}=0$, and similarly, one can show that $a_{2}=a_{3}=0$. Next, if one takes $i=0, j=k=$ $1=1$, one gets $\mathrm{a}_{6}=0$, and $\mathrm{a}_{4}=\mathrm{a}_{5}=0$ are similarly proved. Hence the 6 tensors $(2.1,2.3)$ are indeed linearly independent. Thus in the case $d=2, r=4$, there are 6 linearly independent isotropic tensors forming a complete set, which may be chosen to be $(2.1,2.3)$.

THE CASE $r=6:-$

Here we choose the indices as i, j, k, l, m, n; the possible candidates for linearly independent isotropic tensors, are now

$$
\eta \eta \eta, \eta \eta \epsilon, \eta \epsilon \epsilon, \epsilon \epsilon \epsilon
$$

in the obvious short hand notation introduced earlier. However, as identities (4) and others obtained by choosing other sets of 4 indices out of the 6 indices

$$
\mathrm{i}, \mathrm{j}, \mathrm{k}, \mathrm{l}, \mathrm{m}, \mathrm{n} \text {, }
$$

remain valid here, one sees that $\eta \epsilon \epsilon$ can always be expressed as a linear combination of tensors of the form $\eta \eta \eta$, while $\epsilon \epsilon \epsilon$ can be expressed as a linear combination of tensors of the form $\eta \eta \epsilon$. It follows that in order to get all the linearly independent isotropic tensors of rank 6 , we need to consider only those which are of the form

\section{$\eta \eta \eta$ and $\eta \eta \epsilon$}

we consider these one by one.

The total number of isotropic tensors of the form

$$
\eta \eta \eta \equiv \eta_{i j} \eta_{k l} \eta_{m n}
$$

is

$$
\frac{6 !}{2 ! 2 ! 2 ! 3 !}=15
$$

we write them explicitly as follows.

$$
\begin{aligned}
& \eta_{i j} \eta_{k l} \eta_{m n}, \eta_{i j} \eta_{k m} \eta_{l n}, \eta_{i j} \eta_{k n} \eta_{l m}, \\
& \eta_{i k} \eta_{j l} \eta_{m n}, \eta_{i k} \eta_{j m} \eta_{l n}, \eta_{i k} \eta_{j n} \eta_{l m}, \\
& \eta_{i l} \eta_{j k} \eta_{m n}, \eta_{i l} \eta_{j m} \eta_{k n}, \eta_{i l} \eta_{j n} \eta_{k m}, \\
& \eta_{i m} \eta_{j k} \eta_{l n}, \eta_{i m} \eta_{j l} \eta_{k n}, \eta_{i m} \eta_{j n} \eta_{k l}, \\
& \eta_{i n} \eta_{j k} \eta_{l m}, \eta_{i n} \eta_{j l} \eta_{k m}, \eta_{i n} \eta_{j m} \eta_{k l} .
\end{aligned}
$$

On the other hand, the total number of tensors of the form

$$
\eta \eta \epsilon \equiv \eta_{i j} \eta_{k l} \epsilon_{m n}
$$

is

$$
\frac{6 !}{2 ! 2 ! 2 ! 2 !}=45
$$

explicitly, these are

$$
\begin{gathered}
\left\{\begin{array}{l}
\eta_{i j} \eta_{k l} \epsilon_{m n}, \eta_{i k} \eta_{j l} \epsilon_{m n}, \eta_{i l} \eta_{j k} \epsilon_{m n} \\
\eta_{i j} \eta_{k m} \epsilon_{l n}, \eta_{i k} \eta_{j m} \epsilon_{l n}, \eta_{i m} \eta_{j k} \epsilon_{l n} \\
\eta_{i j} \eta_{l m} \epsilon_{k n}, \eta_{i l} \eta_{j m} \epsilon_{k n}, \eta_{i m} \eta_{j l} \epsilon_{k n} \\
\eta_{i k} \eta_{l m} \epsilon_{j n}, \eta_{i l} \eta_{k m} \epsilon_{j n}, \eta_{i m} \eta_{k l} \epsilon_{j n} \\
\eta_{j k} \eta_{l m} \epsilon_{i n}, \eta_{j l} \eta_{k m} \epsilon_{i n}, \eta_{j m} \eta_{k l} \epsilon_{i n}
\end{array}\right\} \\
\left\{\begin{array}{l}
\eta_{i j} \eta_{k n} \epsilon_{l m}, \eta_{i k} \eta_{j n} \epsilon_{l m}, \eta_{i n} \eta_{j k} \epsilon_{l m} \\
\eta_{i j} \eta_{l n} \epsilon_{k m}, \eta_{i l} \eta_{j n} \epsilon_{k m}, \eta_{i n} \eta_{j l} \epsilon_{k m} \\
\eta_{i k} \eta_{l n} \epsilon_{j m}, \eta_{i l} \eta_{k n} \epsilon_{j m}, \eta_{i n} \eta_{k l} \epsilon_{j m} \\
\eta_{j k} \eta_{l n} \epsilon_{i m}, \eta_{j l} \eta_{k n} \epsilon_{i m}, \eta_{j n} \eta_{k l} \epsilon_{i m}
\end{array}\right\} \epsilon_{\bullet m}, \\
\left\{\begin{array}{l}
\eta_{i j} \eta_{m n} \epsilon_{k l}, \eta_{i m} \eta_{j l} \epsilon_{k l}, \eta_{i n} \eta_{j m} \epsilon_{k l} \\
\eta_{i k} \eta_{m n} \epsilon_{j l}, \eta_{i m} \eta_{k n} \epsilon_{j l}, \eta_{i n} \eta_{k m} \epsilon_{j l} \\
\eta_{j k} \eta_{m n} \epsilon_{i l}, \eta_{j m} \eta_{k n} \epsilon_{i l}, \eta_{j n} \eta_{k m} \epsilon_{i l}
\end{array}\right\} \epsilon_{\bullet l}, \\
\left\{\begin{array}{l}
\eta_{i l} \eta_{m n} \epsilon_{j k}, \eta_{i m} \eta_{l n} \epsilon_{j k}, \eta_{i n} \eta_{l m} \epsilon_{j k} \\
\eta_{j l} \eta_{m n} \epsilon_{i k}, \eta_{j m} \eta_{l n} \epsilon_{i k}, \eta_{j n} \eta_{l m} \epsilon_{i k}
\end{array}\right\} \epsilon_{\bullet k}, \\
\left\{\begin{array}{l}
\left.\eta_{k l} \eta_{m n} \epsilon_{i j}, \eta_{k m} \eta_{l n} \epsilon_{i j}, \eta_{k n} \eta_{l m} \epsilon_{i j}\right\} \epsilon_{\bullet j} .
\end{array}\right.
\end{gathered}
$$

With the help of a technique used by Rashid et. al. in [12] to obtain the number of linearly independent isotropic tensors of rank 6 under $\mathrm{SO}(2)$, it is possible to show that there exist just 20 linearly independent tensors among these 60 , and that 10 of them can be taken from the 15 tensors of the form $\eta \eta \eta$ while the other 10 can be taken from the 45 tensors of the form $\eta \eta \epsilon$. Let us write a linear equation

$$
a_{1} \eta_{i j} \eta_{k l} \eta_{m n}+\ldots+a_{60} \eta_{k n} \eta_{l m} \epsilon_{i j}=0
$$

we then obtain 15 equations between $\mathrm{a}_{1}$ to $\mathrm{a}_{15}$ as follows. Agreeing that

" i j "means

$$
\mathrm{i}=\mathrm{j}=0, \mathrm{k}=\mathrm{l}=\mathrm{m}=\mathrm{n}=1 \text {, }
$$

one gets

$$
\begin{gathered}
\mathrm{ij} \Rightarrow \mathrm{a}_{1}+\mathrm{a}_{2}+\mathrm{a}_{3}=0, \\
\mathrm{ik} \Rightarrow \mathrm{a}_{4}+\mathrm{a}_{5}+\mathrm{a}_{6}=0, \\
\mathrm{il} \Rightarrow \mathrm{a}_{7}+\mathrm{a}_{8}+\mathrm{a}_{9}=0, \\
\mathrm{im} \Rightarrow \mathrm{a}_{10}+\mathrm{a}_{11}+\mathrm{a}_{12}=0, \\
\mathrm{in} \Rightarrow \mathrm{a}_{13}+\mathrm{a}_{14}+\mathrm{a}_{15}=0, \\
\mathrm{jk} \Rightarrow \mathrm{a}_{7}+\mathrm{a}_{10}+\mathrm{a}_{13}=0, \\
\mathrm{jl} \Rightarrow \mathrm{a}_{4}+\mathrm{a}_{11}+\mathrm{a}_{14}=0, \\
\mathrm{jm} \Rightarrow \mathrm{a}_{5}+\mathrm{a}_{8}+\mathrm{a}_{15}=0, \\
\mathrm{jn} \Rightarrow \mathrm{a}_{6}+\mathrm{a}_{9}+\mathrm{a}_{12}=0, \\
\mathrm{kl} \Rightarrow \mathrm{a}_{1}+\mathrm{a}_{12}+\mathrm{a}_{15}=0, \\
\mathrm{~km} \Rightarrow \mathrm{a}_{2}+\mathrm{a}_{9}+\mathrm{a}_{14}=0, \\
\mathrm{kn} \Rightarrow \mathrm{a}_{3}+\mathrm{a}_{8}+\mathrm{a}_{11}=0, \\
\operatorname{lm} \Rightarrow \mathrm{a}_{3}+\mathrm{a}_{6}+\mathrm{a}_{13}=0 \\
\ln \Rightarrow \mathrm{a}_{2}+\mathrm{a}_{5}+\mathrm{a}_{10}=0, \\
\mathrm{mn} \Rightarrow \mathrm{a}_{1}+\mathrm{a}_{4}+\mathrm{a}_{7}=0
\end{gathered}
$$

Now, equation $(5.3) \Rightarrow$

$$
\mathrm{a}_{10}=-\mathrm{a}_{11}-\mathrm{a}_{12}
$$


equation $(5.4) \Rightarrow$

$$
\mathrm{a}_{15}=-\mathrm{a}_{13}-\mathrm{a}_{14}
$$

equation $(5.5) \Rightarrow$

$$
a_{7}=-a_{10}-a_{13}=a_{11}+a_{12}-a_{13},
$$

equation $(5.6) \Rightarrow$

$$
\mathrm{a}_{4}=-\mathrm{a}_{11}-\mathrm{a}_{14}
$$

equation $(5.10) \Rightarrow$

$$
\mathrm{a}_{2}=-\mathrm{a}_{9}-\mathrm{a}_{14}
$$

equation (5.9) $\Rightarrow$

$$
a_{1}=-a_{12}-a_{15}=-a_{12}+a_{13}+a_{14}
$$

equation $(5.1) \Rightarrow$

$$
a_{3}=-a_{1}-a_{2}=a_{12}-a_{13}-a_{14}+a_{9}+a_{14}=a_{9}+a_{12}-a_{13},
$$

equation (5.2) $\Rightarrow$

$$
a_{8}=-a_{7}-a_{9}=-a_{11}-a_{12}+a_{13}-a_{9},
$$

equation $(5.7) \Rightarrow$

$$
\begin{gathered}
a_{5}=-a_{8}-a_{15}=a_{11}+a_{12}-a_{13}+a_{9}+a_{13}+a_{14} \\
=a_{9}+a_{11}+a_{12}+a_{14},
\end{gathered}
$$

equation $(5.8) \Rightarrow$

$$
\mathrm{a}_{6}=-\mathrm{a}_{9}-\mathrm{a}_{12}
$$

These can be written systematically as

$$
\begin{gathered}
\mathrm{a}_{1}=-\mathrm{a}_{12}+\mathrm{a}_{13}+\mathrm{a}_{14}, \\
\mathrm{a}_{2}=-\mathrm{a}_{9}-\mathrm{a}_{14}, \\
\mathrm{a}_{3}=\mathrm{a}_{9}+\mathrm{a}_{12}-\mathrm{a}_{13}, \\
\mathrm{a}_{4}=-\mathrm{a}_{11}-\mathrm{a}_{14}, \\
\mathrm{a}_{5}=\mathrm{a}_{9}+\mathrm{a}_{11}+\mathrm{a}_{12}+\mathrm{a}_{14}, \\
\mathrm{a}_{6}=-\mathrm{a}_{9}-\mathrm{a}_{12}, \\
\mathrm{a}_{7}=\mathrm{a}_{11}+\mathrm{a}_{12}-\mathrm{a}_{13}, \\
\mathrm{a}_{8}=-\mathrm{a}_{9}-\mathrm{a}_{11}-\mathrm{a}_{12}+\mathrm{a}_{13}, \\
\mathrm{a}_{10}=-\mathrm{a}_{11}-\mathrm{a}_{12}, \\
\mathrm{a}_{15}=-\mathrm{a}_{13}-\mathrm{a}_{14} .
\end{gathered}
$$

Hence if one considers the linear equation

$$
a_{1} \eta_{i j} \eta_{k l} \eta_{m n}+\ldots+a_{15} \eta_{i n} \eta_{j m} \eta_{k l}=0
$$

then it follows from equations (6) that the 10 a's

$$
a_{1}, a_{2}, a_{3}, \ldots a_{8}, a_{10}, a_{15}
$$

vanish when

$$
\mathrm{a}_{9}=\mathrm{a}_{11}=\mathrm{a}_{12}=\mathrm{a}_{13}=\mathrm{a}_{14}=0
$$

this implies that the 10 isotropic tensors of which the a's of (8) are the coefficient, are linearly independent. Further, if any one of the above 5 a's is nonzero, then some of the a's of (8) will also be nonzero, so that equation (7) will show that each of the isotropic tensor corresponding to one of the above 5 a's is a linear combination of those corresponding to a's of (8). It follows that among the 15 isotropic tensors of the form $\eta \eta \eta$, precisely 10 are linearly independent and the rest are linear combination of these 10 .

Next, consider the tensors of the form $\eta \eta \epsilon$. Using the statement given on lines 8-10 of first column of page 3 , with 1 replaced by $n$ and i, j, k, replaced by i, j, k, l, m, we see that all the tensors of the form

$$
\eta \eta \epsilon_{\bullet m}, \eta \eta \epsilon_{\bullet l}, \eta \eta \epsilon_{\bullet k}, \eta \eta \epsilon_{\bullet j}
$$

can be expressed in terms of the 15 tensors of the form $\eta \eta \epsilon_{\bullet n}$. It follows that among the 45 tensors of the form $\eta \eta \epsilon$, all the linearly independent ones may be chosen from those of the type $\eta \eta \epsilon_{\bullet n}$. To find these linearly independent tensors, consider the equation

$$
a_{1} \eta_{i j} \eta_{k l} \epsilon_{m n}+\cdots+a_{15} \eta_{j m} \eta_{k l} \epsilon_{i n}=0
$$

Again agreeing that " $\mathrm{i} j \mathrm{k}$ " means

$$
\mathrm{i}=\mathrm{j}=\mathrm{k}=0, \mathrm{l}=\mathrm{m}=\mathrm{n}=1,
$$

we see that the above equation leads to the following 10 equations among the $a_{j}$ :

$$
\left\{\begin{array}{c}
i j k \Rightarrow a_{13}+a_{10}+a_{7}=0, \\
i j l \Rightarrow a_{14}+a_{11}+a_{4}=0, \\
i j m \Rightarrow a_{15}+a_{12}+a_{1}=0, \\
i k l \Rightarrow a_{5}+a_{8}+a_{15}=0, \\
i k m \Rightarrow a_{2}+a_{9}+a_{14}=0, \\
i l m \Rightarrow a_{3}+a_{6}+a_{13}=0, \\
j k l \Rightarrow a_{6}+a_{9}+a_{12}=0, \\
j k m \Rightarrow a_{3}+a_{8}+a_{11}=0, \\
j l m \Rightarrow a_{2}+a_{5}+a_{10}=0, \\
k l m \Rightarrow a_{1}+a_{4}+a_{7}=0 .
\end{array}\right\}
$$

Now

$$
\begin{gathered}
(9.2) \Rightarrow a_{4}=-a_{11}-a_{14}, \\
(9.5) \Rightarrow a_{2}=-a_{9}-a_{14} \\
(9.7) \Rightarrow a_{6}=-a_{9}-a_{12} \\
(9.6) \Rightarrow a_{3}=-a_{6}-a_{13}=a_{9}+a_{12}-a_{13}, \\
(9.8) \Rightarrow a_{8}=-a_{3}-a_{11}=-a_{9}-a_{11}-a_{12}+a_{13},
\end{gathered}
$$

while

$$
\begin{gathered}
(9.1) \Rightarrow a_{7}+a_{10}=-a_{13}, \\
(9.3) \Rightarrow a_{1}+a_{15}=-a_{12}, \\
(9.4) \Rightarrow a_{5}+a_{15}=-a_{8}=a_{9}+a_{11}+a_{12}-a_{13}, \\
(9.9) \Rightarrow a_{5}+a_{10}=-a_{2}=a_{9}+a_{14}, \\
(9.10) \Rightarrow a_{1}+a_{7}=-a_{4}=a_{11}+a_{14} .
\end{gathered}
$$

From the last 5 equations, we get 


$$
\begin{array}{cc}
a_{10}+a_{15} \equiv\left(a_{7}+a_{10}\right)-\left(a_{1}+a_{7}\right)+\left(a_{1}+a_{15}\right) & a_{10}-a_{15} \equiv\left(a_{5}+a_{10}\right)-\left(a_{5}+a_{15}\right) \\
=-a_{13}-a_{11}-a_{14}-a_{12} & =a_{9}+a_{14}-a_{9}-a_{12}+a_{13}-a_{11} \\
\Rightarrow a_{10}=\frac{1}{2}\left(-a_{13}-a_{11}-a_{14}-a_{12}+a_{14}-a_{12}+a_{13}-a_{11}\right)=-a_{11}-a_{12} & =a_{14}-a_{12}+a_{13}-a_{11}
\end{array}
$$

Hence

$$
\begin{gathered}
a_{1}=-a_{15}-a_{12}=a_{13}+a_{14}-a_{12}, \\
a_{7}=-a_{10}-a_{13}=a_{11}+a_{12}-a_{13}, \\
a_{5}=-a_{10}+a_{9}+a_{14}=a_{11}+a_{12}+a_{9}+a_{14} .
\end{gathered}
$$

Collecting the values of

$$
a_{1}-a_{8}, a_{10}, a_{15}
$$

we get

$$
\begin{gathered}
a_{1}=-a_{12}+a_{13}+a_{14}, \\
a_{2}=-a_{9}-a_{14}, \\
a_{3}=a_{9}+a_{12}-a_{13}, \\
a_{4}=-a_{11}-a_{14}, \\
a_{5}=a_{9}+a_{11}+a_{12}+a_{14}, \\
a_{6}=-a_{9}-a_{12}, \\
a_{7}=a_{11}+a_{12}-a_{13}, \\
a_{8}=-a_{9}-a_{11}-a_{12}+a_{13}, \\
a_{10}=-a_{11}-a_{12}, \\
a_{15}=-a_{13}-a_{14},
\end{gathered}
$$

It is interesting to note that these are identical with equations (6) obtained earlier. Applying now the same arguments as were used after equation (6), we conclude that out of the 15 isotropic tensors of the form $\eta \eta \epsilon_{\bullet}$, precisely 10 are linearly independent and these can be chosen to be the ones whose coefficients are $a_{1}-a_{8}, a_{10}, a_{15}$ in the above linear equation.

We have thus completed the proof of our assertion that out of the 60 isotropic tensors of rank 6 of the form

$\eta \eta \eta, \eta \eta \epsilon$,

precisely 20 are linearly independent (and form a complete set) and that 10 of these may be chosen to be of the form $\eta \eta \eta$ while the rest of 10 can be chosen to be of the form $\eta \eta \epsilon_{\bullet}$.

\section{3. $d=3$; Isotropic Tensors Under \\ $\operatorname{SO}(2,1)$}

THE CASE $r=2$. Here, there is only one isotropic tensor, namely $\eta_{i j}$.

THE CASE $r=3$. Here again, there is only one isotropic tensor namely $\epsilon_{i j k}$.

THE CASE $r=4$. Here the possible isotropic tensors are

$$
\eta_{i j} \eta_{k l}, \eta_{i k} \eta_{j l}, \eta_{i l} \eta_{j k}
$$

These are easily checked, as in the case $\mathrm{d}=2$, to be linearly independent; thus there are just 3 linearly independent isotropic tensors of rank 4.

THE CASE $r=5$. Here, the possible isotropic tensors are

$$
\eta_{i j} \epsilon_{k l m}, \eta_{i k} \epsilon_{j l m}, \ldots
$$

Their number is

$$
\frac{5 !}{2 ! 3 !}=\frac{5.4}{2}=10
$$

but we will show that only 6 of these are linearly independent.

From (3), we get

$$
\begin{gathered}
\epsilon_{i j k l} \epsilon_{p q r s}=\left|\begin{array}{c}
\eta_{i p} \eta_{i q} \eta_{i r} \eta_{i s} \\
\eta_{j p} \eta_{j q} \eta_{j r} \eta_{j s} \\
\eta_{k p} \eta_{k q} \eta_{k r} \eta_{k s} \\
\eta_{l p} \eta_{l q} \eta_{l r} \eta_{l s}
\end{array}\right| \\
=\eta_{i p} \epsilon_{j k l} \epsilon_{q r s}-\eta_{i q} \epsilon_{j k l} \epsilon_{p r s}+\eta_{i r} \epsilon_{j k l} \epsilon_{p q s}-\eta_{i s} \epsilon_{j k l} \epsilon_{p q r} \\
=\epsilon_{j k l}\left(\eta_{i p} \epsilon_{q r s}-\eta_{i q} \epsilon_{p r s}+\eta_{i r} \epsilon_{p q s}-\eta_{i s} \epsilon_{p q r}\right) . \\
\text { As } \epsilon_{i j k l}=0 \text { for } \mathrm{d}=3, \text { this gives } \\
\eta_{i p} \epsilon_{q r s}-\eta_{i q} \epsilon_{p r s}+\eta_{i r} \epsilon_{p q s}-\eta_{i s} \epsilon_{p q r}=0 .
\end{gathered}
$$

Choose now

$$
\mathrm{p}=\mathrm{j}, \mathrm{q}=\mathrm{k}, \mathrm{r}=1, \mathrm{~s}=\mathrm{m}
$$

to get

$$
\begin{gathered}
\eta_{i j} \epsilon_{k l m}-\eta_{i k} \epsilon_{j l m}+\eta_{i l} \epsilon_{j k m}-\eta_{i m} \epsilon_{j k l}=0 \\
\Rightarrow \eta_{i m} \epsilon_{j k l}=\eta_{i j} \epsilon_{k l m}-\eta_{i k} \epsilon_{j l m}+\eta_{i l} \epsilon_{j k m} .
\end{gathered}
$$

This shows that $\eta_{i m} \epsilon_{j k l}$ can be expressed as a linear combination of tensors in which the subscripts of $\eta$, do not contain $\mathrm{m}$. As the same will obviously be true for the 3 tensors

$$
\eta_{j m} \epsilon_{i k l}, \eta_{k m} \epsilon_{i j l}, \eta_{l m} \epsilon_{i j k}
$$

we conclude that each of the above 4 tensors can be expressed as a linear combination of the 6 tensors

$$
\eta_{i j} \epsilon_{k l m}, \eta_{i k} \epsilon_{j l m}, \eta_{i l} \epsilon_{j k m}, \eta_{j k} \epsilon_{i l m}, \eta_{j l} \epsilon_{i k m}, \eta_{k l} \epsilon_{i j m}
$$

The linear independence of these 6 tensors is easily checked by writing an equation

$$
a_{1} \eta_{i j} \epsilon_{k l m}+\ldots+a_{6} \eta_{k l} \epsilon_{i j m}=0
$$


and choosing

$$
\mathrm{i}=\mathrm{j}=\mathrm{m}=0, \mathrm{k}=1, \mathrm{l}=2,
$$

which gives $a_{1}=0$; other a's can be shown to be 0 in the same way. Thus, we have shown that there exist precisely 6 linearly independent isotropic tensors of rank 5, again forming a complete set, under $\mathrm{SO}(2,1)$, as clamed earlier.

THE CASE $r=6$ :- Here the possible candidates for the isotropic tensors are

$$
\eta_{i j} \eta_{k l} \eta_{m n}, \epsilon_{i j k} \epsilon_{l m n}
$$

However, we prove in the Appendix B that

$$
\epsilon_{i j k} \epsilon_{l m n}=\left|\begin{array}{l}
\eta_{i l} \eta_{i m} \eta_{i n} \\
\eta_{j l} \eta_{j m} \eta_{j n} \\
\eta_{k l} \eta_{k m} \eta_{k n}
\end{array}\right|
$$

$=$ a linear combination of tensors of the form $\eta_{i j} \eta_{k} \eta_{m} n$, so that one needs to consider only the tensors of the form

$$
\eta_{i j} \eta_{k l} \eta_{m n}
$$

The number of these tensors is

$$
\frac{6 !}{2 ! 2 ! 2 ! 3 !}=\frac{6.5 .4}{8}=15 \text {, }
$$

and they are

$$
\begin{aligned}
& \eta_{i j} \eta_{k l} \eta_{m n}, \eta_{i j} \eta_{k m} \eta_{l n}, \eta_{i j} \eta_{k n} \eta_{l m}, \eta_{i k} \eta_{j l} \eta_{m n}, \eta_{i k} \eta_{j m} \eta_{l n}, \\
& \eta_{i k} \eta_{j n} \eta_{l m}, \eta_{i l} \eta_{j k} \eta_{m n}, \eta_{i l} \eta_{j m} \eta_{k n}, \eta_{i l} \eta_{j n} \eta_{k m}, \eta_{i m} \eta_{j k} \eta_{l n}, \\
& \eta_{i m} \eta_{j l} \eta_{k n}, \eta_{i m} \eta_{j n} \eta_{k l}, \eta_{i n} \eta_{j k} \eta_{l m}, \eta_{i n} \eta_{j l} \eta_{k m}, \eta_{i n} \eta_{j m} \eta_{k l}
\end{aligned}
$$

These can easily checked to be linearly independent, for if there is a linear relation

$$
a_{1} \eta_{i j} \eta_{k l} \eta_{m n}+a_{2} \eta_{i j} \eta_{k m} \eta_{l n}+\ldots+a_{15} \eta_{i n} \eta_{j m} \eta_{k l}=0,
$$

choosing

$$
\mathrm{i}=\mathrm{j}=0, \mathrm{k}=1=1, \mathrm{~m}=\mathrm{n}=2,
$$

one gets $\mathrm{a}_{1}=0$, and one can similarly show that the rest of them are also 0 . This proves the linear independence of the set so that we conclude that there exist precisely 15 linearly independent isotropic tensors of rank 6 , forming a complete set, under $\mathrm{SO}(2,1)$.

\section{4. $d=4$ : Isotropic Tensors Under $\operatorname{SO}(3,1)$ and $\operatorname{SO}(2,2)$}

Here, the matrix $\eta$ is given by

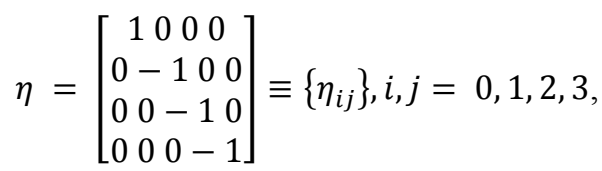

for $\operatorname{SO}(3,1)$, and by

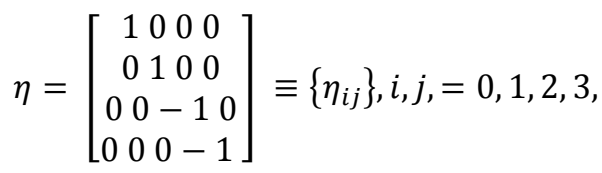

for $\operatorname{SO}(2,2)$; both these cases can be considered together as the arguments are independent of the difference between the two $\eta^{\prime} s$.

THE CASE $r=2$ :

As in the case of $\mathrm{SO}(2,1)$, the only isotropic tensor of rank 2 , in the present case, is $\eta_{i j}$.

THE CASE $r=3$ :

As already mentioned, there are no isotropic tensors of odd rank when $\mathrm{d}=4$.

THE CASE $r=4$ :

Here, the possible candidates for the linearly independent isotropic tensors are

$$
\eta_{i j} \eta_{k l}, \eta_{i k} \eta_{j l}, \eta_{i l} \eta_{j k}, \epsilon_{i j k l}
$$

It is easy to check that these are all independent; for, if

$$
a_{1} \eta_{i j} \eta_{k l}+++a_{4} \epsilon_{i j k l}=0
$$

setting

$$
\mathrm{i}=\mathrm{j}=0, \mathrm{k}=1=1 \text {, }
$$

one gets $\mathrm{a}_{1}=0$, and one similarly gets $\mathrm{a}_{2}=\mathrm{a}_{3}=0$. Next, letting

$$
\mathrm{i}=0, \mathrm{j}=1, \mathrm{k}=2, \mathrm{l}=3,
$$

one gets $\mathrm{a}_{4}=0$ i.e. all the 4 tensors are linearly independent.

THE CASE $r=6$ :

Here, the possible candidates for linearly independent tensors are

$$
\frac{6 !}{2 ! 2 ! 2 ! 3 !}=\frac{6.5 .4}{2.2 .2}=15
$$

tensors of the form $\eta \eta \eta$, namely

$$
\begin{aligned}
& \eta_{i j} \eta_{k l} \eta_{m n}, \eta_{i j} \eta_{k m} \eta_{l n}, \eta_{i j} \eta_{k n} \eta_{l m}, \eta_{i k} \eta_{j l} \eta_{m n}, \eta_{i k} \eta_{j m} \eta_{l n}, \\
& \eta_{i k} \eta_{j n} \eta_{l m}, \eta_{i l} \eta_{j k} \eta_{m n}, \eta_{i l} \eta_{j m} \eta_{k n}, \eta_{i l} \eta_{j n} \eta_{k m}, \eta_{i m} \eta_{j k} \eta_{l n}, \\
& \eta_{i m} \eta_{j l} \eta_{k n}, \eta_{i m} \eta_{j n} \eta_{k l}, \eta_{i n} \eta_{j k} \eta_{l m}, \eta_{i n} \eta_{j l} \eta_{k m}, \eta_{i n} \eta_{j m} \eta_{k l},
\end{aligned}
$$
and

$$
\frac{6 !}{2 ! 4 !}=\frac{6.5}{2}=15
$$

tensors of the form $\eta \epsilon$, namely

$$
\begin{aligned}
& \eta_{i j} \epsilon_{k l m n}, \eta_{i k} \epsilon_{j l m n}, \eta_{i l} \epsilon_{j k m n}, \eta_{i m} \epsilon_{j k l n}, \eta_{i n} \epsilon_{j k l m}, \\
& \eta_{j k} \epsilon_{i l m n}, \eta_{j l} \epsilon_{i k m n}, \eta_{j m} \epsilon_{i k l n}, \eta_{j n} \epsilon_{i k l m}, \eta_{k l} \epsilon_{i j m n}, \\
& \eta_{k m} \epsilon_{i j l n}, \eta_{k n} \epsilon_{i j l m}, \eta_{l m} \epsilon_{i j k n}, \eta_{l n} \epsilon_{i j k m}, \eta_{m n} \epsilon_{i j k l} .
\end{aligned}
$$

We now show that all the 15 tensors of the form $\eta \eta \eta$ are 
linearly independent while precisely 10 of the 15 tensors of the form $\eta \epsilon$ are so, so that, in all, there exist 25 linearly independent isotropic tensors of rank 6, which form a complete set.

Consider an equation of the form

$$
a_{1} \eta_{i j} \eta_{k l} \eta_{m n}+\ldots+a_{15} \eta_{i n} \eta_{j m} \eta_{k l}=0
$$

Choosing

$$
\mathrm{i}=\mathrm{j}=0, \mathrm{k}=\mathrm{l}=1, \mathrm{~m}=\mathrm{n}=2,
$$

the above equation gives $\mathrm{a}_{1}=0$; the rest of the a's can be similarly proved to be equal to 0 , so that one may conclude that all the tensors of the form $\eta \eta \eta$ are indeed linearly independent.

Next, to consider the linear independence of tensors of the form $\eta \epsilon$, we see that from Appendix B, we get

$$
\begin{gathered}
\epsilon_{i j k l m} \epsilon_{p q r s t} \\
=(-1)^{4}\left|\begin{array}{c}
\eta_{i p} \eta_{i q} \eta_{i r} \eta_{i s} \eta_{i t} \\
\eta_{j p} \eta_{j q} \eta_{j r} \eta_{j s} \eta_{j t} \\
\eta_{k p} \eta_{k q} \eta_{k r} \eta_{k s} \eta_{k t} \\
\eta_{l p} \eta_{l q} \eta_{l r} \eta_{l s} \eta_{l t} \\
\eta_{m p} \eta_{m q} \eta_{m r} \eta_{m s} \eta_{m t}
\end{array}\right| \\
=-\left[\eta_{i p} \epsilon_{j k l m} \epsilon_{q r s t}-\eta_{i q} \epsilon_{j k l m} \epsilon_{p r s t}+\eta_{i r} \epsilon_{j k l m} \epsilon_{p q s t}-\right. \\
\left.\eta_{i s} \epsilon_{j k l m} \epsilon_{p q r t}+\eta_{i t} \epsilon_{j k l m} \epsilon_{p q r s}\right] \\
=-\epsilon_{j k l m}\left[\eta_{i p} \epsilon_{q r s t}-\eta_{i q} \epsilon_{p r s t}+\eta_{i r} \epsilon_{p q s t}-\eta_{i s} \epsilon_{p q r t}+\right. \\
\left.\eta_{i t} \epsilon_{p q r s}\right] .
\end{gathered}
$$

Hence, for $\mathrm{d}=4$, we will have

$$
\eta_{i p} \epsilon_{q r s t}-\eta_{i q} \epsilon_{p r s t}+\eta_{i r} \epsilon_{p q s t}-\eta_{i s} \epsilon_{p q r t}+\eta_{i t} \epsilon_{p q r s}=0 \text {. }
$$

Choose now

$$
\mathrm{p}=\mathrm{j}, \mathrm{q}=\mathrm{k}, \mathrm{r}=\mathrm{l}, \mathrm{s}=\mathrm{m}, \mathrm{t}=\mathrm{n},
$$

to get

$$
\eta_{i n} \epsilon_{j k l m}=-\eta_{i j} \epsilon_{k l m n}+\eta_{i k} \epsilon_{j l m n}-\eta_{i l} \epsilon_{j k m n}+\eta_{i m} \epsilon_{j k l n}
$$

which shows that $\eta_{i n} \epsilon_{j k l m}$ can be expressed as a linear combination of the tensors

$$
\eta_{i j} \epsilon, \eta_{i k} \epsilon, \eta_{i l} \epsilon, \eta_{i m} \epsilon
$$

One can similarly show that each of the four tensors

$$
\eta_{j n} \epsilon, \eta_{k n} \epsilon, \eta_{l n} \epsilon, \eta_{m n} \epsilon,
$$

can be expressed as a linear combination of tensors of the form

$$
\eta_{a b} \epsilon_{c d e f}
$$

in which $\mathrm{n}$ never appears as an index of $\eta$. It follows that each of the five tensors

$$
\eta_{i n} \epsilon, \eta_{j n} \epsilon, \eta_{k n} \epsilon, \eta_{l n} \epsilon, \eta_{m n} \epsilon
$$

can be expressed as a linear combination of the ten tensors

$$
\eta_{i j} \epsilon, \eta_{i k} \epsilon, \eta_{i l} \epsilon, \eta_{i m} \epsilon, \eta_{j k} \epsilon, \eta_{j l} \epsilon, \eta_{j m} \epsilon, \eta_{k l} \epsilon, \eta_{k m} \epsilon, \eta_{l m} \epsilon .
$$

It is now not difficult to show that these ten tensors are linearly independent. For let

$$
a_{1} \eta_{i j} \epsilon_{k l m n}+a_{2} \eta_{i k} \epsilon_{j l m n}+\ldots+a_{10} \eta_{l m} \epsilon_{i j k n}=0 .
$$

Then the choice

$$
\mathrm{i}=\mathrm{j}=\mathrm{n}=0, \mathrm{k}=1, \mathrm{l}=2, \mathrm{~m}=3,
$$

gives $\mathrm{a}_{1}=0$; all other a's are proved to be equal to zero in exactly the same way. It follows that the ten tensors are indeed linearly independent. Thus we conclude that there are $15+10=25$ linearly independent isotropic tensors of rank 6 , forming a complete set, under $\mathrm{SO}(3,1)$ and $\mathrm{SO}(2,2)$.

\section{Conclusion}

We have obtained all the linearly independent isotropic tensors of ranks from 2 to 6 , under the non-compact rotation groups $\mathrm{SO}(1,1), \mathrm{SO}(2,1), \mathrm{SO}(3,1)$ and $\mathrm{SO}(2,2)$. We find that their number is exactly the same as that of corresponding linearly independent isotropic tensors under the compact rotation groups $\mathrm{SO}(2), \mathrm{SO}(3)$ and $\mathrm{SO}(4)$ as given by Naila [11] and Faiz and Rashid [10, 12].

\section{Appendix A}

If $\left\{a_{i j}\right\}$ is an element of $\operatorname{SL}(3, \mathbb{R})$, then it transforms vectors $x_{i} \in \mathbb{R}^{3}$, to

$$
x_{i}^{\prime}=a_{i j} x_{j}
$$

as $\varepsilon_{i j k}$ is a tensor of $3^{\text {rd }}$ rank, $\left\{a_{i j}\right\}$ will transform it to

$$
\varepsilon_{i j k}^{\prime}=a_{i l} a_{j m} a_{k n} \varepsilon_{l m n} .
$$

Now if $\underline{a}, \underline{b}, \underline{c}$ are 3 row vectors so that

$$
\left[\frac{a}{b}\right]
$$

is a $3 \times 3$ matrix, then we know that

$$
\operatorname{det}\left[\frac{\underline{a}}{\underline{b}}\right]=\varepsilon_{i j k} a_{i} b_{j} c_{k} .
$$

Hence, if for a given matrix $\left\{a_{i j}\right\}$ of $\operatorname{SL}(3, \mathbb{R})$, we set $\underline{a}_{i}$ as its ith row, then

$$
\underline{a}_{i}=\left[\begin{array}{lll}
a_{i 1} & a_{i 2} & a_{i 3}
\end{array}\right] \text { i.e. }\left(\underline{a}_{i}\right)_{l}=a_{i l},
$$

and similarly for $\underline{a}_{j}$ and $\underline{a}_{k}$; we then have

$$
\begin{aligned}
& \varepsilon_{i j k}^{\prime}=a_{i l} a_{j m} a_{k n} \varepsilon_{l m n} \\
= & \varepsilon_{l m n}\left(\underline{a}_{i}\right)_{l}\left(\underline{a}_{j}\right)_{m}\left(\underline{a}_{k}\right)_{n}
\end{aligned}
$$




$$
\begin{gathered}
=\operatorname{det}\left[\begin{array}{l}
\underline{a}_{i} \\
\underline{a}_{j} \\
\underline{a}_{k}
\end{array}\right]=\varepsilon_{i j k} \operatorname{det}\left[\begin{array}{l}
\underline{a}_{1} \\
\underline{a}_{2} \\
\underline{a}_{3}
\end{array}\right]=\varepsilon_{i j k} \operatorname{det}\left\{a_{i j}\right\} \\
\Rightarrow \varepsilon_{i j k}^{\prime}=\varepsilon_{i j k}
\end{gathered}
$$

showing that $\varepsilon_{i j k}$ is indeed isotropic underSL $(3, \mathbb{R})$. In particular, it will be isotropic under both $\mathrm{SO}(3)$ and $\operatorname{SO}(2,1)$ as these are just subgroups of $\operatorname{SL}(3, \mathbb{R})$. In fact, the result holds for arbitrary $n \geq 2$ rather than for just $n=3$; thus we have

$\varepsilon_{i_{1 i_{2} \ldots i_{k}}}$ is an isotropic tensor under $\operatorname{SL}(\mathrm{k}, \mathbb{R}), \mathrm{k} \geq 2$.

The fact that $\varepsilon_{i j}$ is isotropic under both $\mathrm{SO}(2)$ and $\operatorname{SO}(1$, 1) follows directly from this result.

\section{Appendix B}

Let $\mathrm{A} \equiv\left\{a_{i j}\right\} \in S O(n-1,1)$, with

$$
\mathrm{i}, \mathrm{j}=0,1, \ldots, \mathrm{n}-1 \text {, }
$$

and metric in $M(n-1,1)$ given by

$$
x^{2}=x_{0}^{2}-x_{1}^{2}-\ldots-x_{n-1}^{2} .
$$

Then

$$
A=\left[\begin{array}{lll}
a_{0} a_{1} & \cdots & a_{n-1}
\end{array}\right], A^{T}=\left[\begin{array}{c}
a_{0}^{T} \\
a_{1}^{T} \\
\cdot \\
\cdot \\
\cdot \\
a_{n-1}^{T}
\end{array}\right]
$$

where

$$
a_{j}=\left[\begin{array}{c}
a_{0 j} \\
a_{1 j} \\
\cdot \\
\cdot \\
\cdot \\
a_{n-1 j}
\end{array}\right], a_{j}^{T}=\left[\begin{array}{llll}
a_{0 j} a_{1 j} & \cdots & a_{n-1 j}
\end{array}\right]
$$

so that

$$
\left(a_{j}\right)_{i}=a_{i j} \text { and }\left(a_{j}^{T}\right)_{i}=a_{i j} .
$$

Put now

$$
\hat{A}=\eta A=\left[\begin{array}{c}
a_{00} a_{01} \cdots \ldots a_{0 n-1} \\
-a_{10}-a_{11} \ldots . . a_{1 n-1} \\
\cdot \\
\cdot \\
-a_{n-11}-a_{n-12} \cdots-a_{n-1 n-1}
\end{array}\right]
$$

$$
\hat{a}_{j}=\left[\begin{array}{c}
a_{0 j} \\
-a_{1 j} \\
\cdot \\
\cdot \\
-a_{n-1 j}
\end{array}\right]
$$

It can now be noted that

$$
\begin{gathered}
\operatorname{det}\left[\begin{array}{c}
a_{i_{0}}^{T} \\
a_{i_{1}}^{T} \\
\cdot \\
\cdot \\
\cdot \\
a_{i_{n-1}}^{T}
\end{array}\right]=\varepsilon_{i_{0} i_{1} \ldots i_{n-1}} \operatorname{det}\left[\begin{array}{c}
a_{0}^{T} \\
a_{1}^{T} \\
\cdot \\
a_{n-1}^{T}
\end{array}\right] \\
=\varepsilon_{i_{0 i_{1 \ldots i} \ldots i_{n-1}}} \operatorname{det} A^{T}=\varepsilon_{i_{0 i_{1} \ldots i_{n-1}}}, \\
\operatorname{det}\left[\hat{a}_{i_{0}} \hat{a}_{i_{1} \ldots \hat{a}_{i_{n-1}}}\right]=\varepsilon_{i_{0 i_{1} \ldots i_{n-1}}} \operatorname{det}\left[\hat{a}_{0} \hat{a}_{1} \ldots \hat{a}_{n-1}\right] \\
=\varepsilon_{i_{0 i_{1} \ldots i_{n-1}}} \operatorname{det} \hat{A}=\varepsilon_{i_{0 i_{1} \ldots i_{n-1}}}(\operatorname{det} \eta \cdot \operatorname{det} A) \\
=(-1)^{n-1} \varepsilon_{i_{0 i_{1} \ldots i_{n-1}},}
\end{gathered}
$$

$a_{i}^{T} \cdot \hat{a}_{j}$ (matrix multiplication)

$$
\begin{aligned}
& =\left[a_{0 i} a_{1 i} \ldots a_{n-1 i}\right] \cdot\left[\begin{array}{c}
a_{0 j} \\
-a_{1 j} \\
\cdot \\
\cdot \\
\cdot \\
-a_{n-1 j}
\end{array}\right] \\
& =a_{0 i} a_{0 j}-a_{1 i} a_{1 j}-\ldots-a_{n-1 i} a_{n-1 j}
\end{aligned}
$$

$=a_{i} \cdot a_{j}($ scalar multiplication inM $(\mathrm{n}-1,1))$

$$
=\eta_{i j},
$$

so that we get

$$
\epsilon_{i_{0 i_{1 \ldots i} i_{n-1}}} \varepsilon_{j_{0 j_{1} \ldots j_{n-1}}}
$$$$
=\operatorname{det}\left[\begin{array}{c}
a_{i_{0}}^{T} \\
a_{i_{1}}^{T} \\
\cdot \\
\cdot \\
a_{i_{n-1}}^{T}
\end{array}\right] \cdot(-1)^{n-1} \operatorname{det}\left[\hat{a}_{j_{0}} \hat{a}_{j_{1}} \ldots \hat{a}_{j_{n-1}}\right]
$$$$
=(-1)^{n-1} \operatorname{det}\left[\begin{array}{c}
a_{i_{0}}^{T} \\
a_{i_{1}}^{T} \\
\cdot \\
\cdot \\
a_{n-1}^{T}
\end{array}\right] \cdot\left[\hat{a}_{j_{0}} \hat{a}_{j_{1}} \ldots \hat{a}_{j_{n-1}}\right]
$$

with 


$$
=(-1)^{n-1}\left|\begin{array}{c}
\eta_{i_{0 j} j_{0}} \eta_{i_{0 j}} \ldots \eta_{i_{0 j} j_{n-1}} \\
\eta_{i_{1 j_{0}}} \eta_{i_{1 j_{1}}} \ldots \eta_{i_{1 j_{n-1}}} \\
\cdot \\
\cdot \\
\cdot \\
\eta_{i_{n-1} j_{0}} \eta_{i_{n-1} j_{1}} \ldots \eta_{i_{n-1} j_{n-1}}
\end{array}\right|
$$

which is the required equation number (3) mentioned in the text.

\section{References}

[1] Faiz Ahmad and Riaz Ahmad Khan, Eigenvectors of a rotation matrix; Q. Jl. Mech. Appl. Maths., 62, (2009), 297 - 310.

[2] Hirth, J. P. and Lothe, Theory of Dislocations, McGraw-Hill, New York, 1968..

[3] Juretschke, H. J., Crystal Physics, Benjamin, Menlo Park, 1974.

[4] Hearmon, R. F. S., An Introduction to Applied Anisotropic Elasticity; OUP, New York, 1963.

[5] Norris, A. N., Quadratic invariants of elastic moduli; Q. J1. Mech. Appl. Math., 60, (2007), 367 - 389.
[6] Zhou, S., Li, A. and Wang, B., A reformulation of constitutive relations in the strain gradient elasticity theory for isotropic models; Int. J. Solids Struct. 80, 28e37, 2016.

[7] Lazar, M., Irreducible Decomposition of Strain Gradient Tensor in Isotropic Strain Gradient Elasticity. ArXiv preprint, ArXiv: 1604. 07254. 2016.

[8] Itin, Y. and Hehl, F. W., Irreducible decomposition of elasticity tensor under linear and orthogonal groups and their physical consequences, Journal of Physics: Conference Series 597012046, 2015.

[9] Gusev, A. A. and Lurie, S. A.,. Symmetry conditions in strain elasticity, Math. Mech. Solid, 2015, $1 \mathrm{e} 9$. doi.org/10.1177/10812865 15606960 .

[10] Ahmad, F. and Rashid, M. A., Linear Invariants of Cartesian Tensors. Q. Jl. Mech. Appl. Maths., 62, 31 - 38 (2009).

[11] Amir, Naila, Linear Invariants of a Cartisian Tensor under SO(4): M. Phil Dissertation, Centre for Advanced Mathematics and Physics, National University of Science and Technology, Islamabad, Pakistan (2010).

[12] Ahmad, F. and Rashid, M. A., Counting Invariants of a Cartisian Tensor. Paper presented in IMACS World Congress on Computational and Applied Mathematics and Applications in Science and Engineering, held in University of Georgia, Athens, USA, 3 - 5 August, 2009. 